\title{
Overexpression, purification, crystallization and preliminary X-ray crystallographic characterization of the receiver domain of the response regulator PhoP from Enterococcus faecalis ATCC 29212
}

\author{
Yoon Chae Jeong and Ki Seog Lee*
}

\begin{abstract}
Phosphate (Pho) regulon plays a critical role in bacterial phosphate homeostasis. It is regulated by two-component system (TCS) that comprises a sensor histidine kinase and transcriptional response regulator (RR). PhoP from Enterococcus faecalis (EfPhoP) belongs to the OmpR subfamily of RRs. It has not yet been structurally characterized because it is difficult to crystallize it to full-length form. In this study, a truncated form of EfPhoP containing the receiver domain (EfPhoP-RD) was constructed, purified to homogeneity and crystallized using the hanging-drop vapour-diffusion method. The crystal of EfPhoP-RD diffracted to 3.5 A resolution and belonged to the orthorhombic space group $C 222_{1}$, with unit-cell parameters $\mathrm{a}=118.74, \mathrm{~b}=189.83, \mathrm{C}=189.88 \AA$. The asymmetric unit contains approximately 12 molecules, corresponding to a Matthews coefficient $\left(V_{m}\right)$ of $2.50 \AA^{3} \mathrm{Da}^{-1}$ with a solvent content of $50.9 \%$.
\end{abstract}

Keywords: Phosphate (Pho) regulon, Two-component system, Response regulator, PhoP, Enterococcus faecalis

\section{Introduction}

Phosphorous is one of cellular component important for many biological and biochemical processes in living organisms, such as the formations of nucleic acids (DNA and RNA) and membrane phospholipids, post-translational modifications for signal transduction, etc. [1]. The usefulness of phosphorous has been applied to extensive field such as agriculture, medicine and pharmaceuticals. In particular, phosphorous is required to secure a high level of productivity in agriculture [2, 3]. In bacteria, the major form of phosphorous is orthophosphate known as inorganic phosphates $(\mathrm{Pi})$ [4]. Despite the varying importance of $\mathrm{Pi}$ in cellular function, it is usually found at very low concentration in natural environment [5]. Therefore, bacteria and other organisms must have relevant systems

*Correspondence: kslee@cup.ac.kr

Department of Clinical Laboratory Science, College of Health Sciences, Catholic University of Pusan, Busan 46252, Republic of Korea that include physiological and biochemical responses to overcome the deficiency of this nutrient [6].

A unique mechanism associated with the maintenance of $\mathrm{Pi}$ in bacteria as a regulatory circuit is the phosphate (Pho) regulon that is regulated by a two-component system (TCS) $[5,6]$. The Pho regulon is one of the most rational and effective regulatory mechanisms. It is wellstudied in model cells such as Escherichia coli [7] and Bacillus subtilis [8]. Later, it has been characterized in many other bacterial species [9]. TCSs are signal transduction pathways commonly used by bacteria to recognize and adapt to stimuli caused by environmental changes. TCS consists of histidine kinase (HK) as an inner-membrane sensor kinase that recognizes several specific environmental signals and the transcriptional response regulator (RR) protein that mediates cellular responses by regulating expression of specific genes or modulating protein functions in the cytoplasm [10]. Although these proteins are known by different names in some bacteria $[11,12]$, upon Pi deficiency, the RR is 
phosphorylated by the HK. Thereby, the phosphorylated RR can bind to specific DNA sequences and then activate or suppress the transcription of their corresponding genes $[13,14]$.

A number of new members of Pho regulon have been identified from several bacteria in past years, but there still remain numerous undiscovered questions such as the detailed function of the entire system and the mechanisms connecting the Pho regulon to pathogenesis [5]. Among them, enterococci are normal flora in human intestine of healthy adults, but also they are one of the major causes of hospital infections that leads a variety of diseases, including bacteremia, urinary tract and central nervous system infections [15]. Most clinical isolates of enterococci are Enterococcus faecalis along with Enterococcus faecium [16]. However, the TCS related to the Pho regulon in this strain has not been well studied except for the VanRS system that regulates the resistance of enterococci to vancomycin [17].

Most RRs have two distinct domains involving the receiver domain of $\mathrm{N}$-terminus and the effector domain of $\mathrm{C}$-terminus. On the basis of the structure and function of the effector domains, they can be classified into subfamilies [14]. PhoP belongs to the OmpR/PhoB subfamily

Table 1 Macromolecule-production information

\begin{tabular}{|c|c|}
\hline Source organism & Enterococcus faecalis ATCC 29212 \\
\hline DNA source & Genomic DNA \\
\hline Forward primer ${ }^{a}$ & 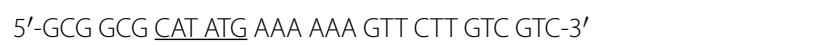 \\
\hline Reverse primer ${ }^{a}$ & 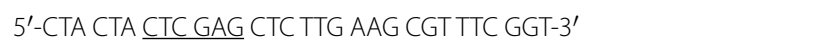 \\
\hline Cloning vector & pET-28a \\
\hline Expression vector & pET-28a \\
\hline Expression host & E. coli BL21 (DE3) \\
\hline $\begin{array}{l}\text { Complete amino-acid sequence } \\
\text { of the construct produced }\end{array}$ & $\begin{array}{l}\text { MGSSHHHHHHSSGLVPRGSHMKKVLVVDDEPSILTLLTFNLEKEGYQVTTSENG- } \\
\text { KNGFELALSNQYDFIILDVMLPGMDGLEITKALRREKIDTPILILTAKDEQVDKII- } \\
\text { GLEIGADDYLTKPFSPREVLARMKAIFRRLKPTTTETLQELEHHHHHH }\end{array}$ \\
\hline
\end{tabular}

a Restriction enzyme sites are underlined

b The extra amino acids introduced into the EfPhoP-RD protein by cloning are underlined

Table 2 Data-collection statistics

\begin{tabular}{|c|c|c|}
\hline & \multicolumn{2}{|l|}{ EfPhoP-RD } \\
\hline & Thick plate-shape & Thin rod-shape \\
\hline Diffraction source & Beamline 7A, PAL & \\
\hline Wavelength $(\AA)$ & 1.0000 & \\
\hline Detector & ADSC Quantum 270r CCD & \\
\hline Crystal-to-detector distance (mm) & 450 & 350 \\
\hline Rotation range per image $\left({ }^{\circ}\right)$ & 1 & 1 \\
\hline Total rotation range $\left({ }^{\circ}\right)$ & 180 & 360 \\
\hline Exposure time per image (s) & 5 & 2 \\
\hline Space group & $P 2,2,2_{1}$ & $\mathrm{C} 222_{1}$ \\
\hline Unit-cell parameters $\left(\AA,{ }^{\circ}\right)$ & $\begin{array}{l}a=188.63, b=187.71, c=197.49 \\
a=\beta=\gamma=90\end{array}$ & $\begin{array}{l}a=118.74, b=189.83, c=189.88 \\
a=\beta=\gamma=90\end{array}$ \\
\hline Resolution range (Å) & $50.0-5.0(5.18-5.00)$ & $50.0-3.5(3.63-3.50)$ \\
\hline Total no. of reflections & 628,425 & $1,931,024$ \\
\hline No. of unique reflections & 26,755 & 20,764 \\
\hline Completeness (\%) & $87.1(81.2)$ & $75.4(65.1)$ \\
\hline Multiplicity & $3.0(1.9)$ & $2.5(1.7)$ \\
\hline$<1 / \sigma(I)>$ & $10.8(2.0)$ & $6.6(2.4)$ \\
\hline $\mathrm{CC}_{1 / 2}$ & $0.994(0.172)$ & $0.973(0.334)$ \\
\hline$R_{\text {merge }}^{a}(\%)$ & $10.1(35.1)$ & $14.3(34.9)$ \\
\hline
\end{tabular}

Values in parentheses are for the outer shell

${ }^{a} R_{\text {merge }}=\sum_{h k l i} I_{h k l i}-<I_{h k l}>\mid / \sum_{h k l i} I_{h k l i}$ where $/$ represents the observed intensity, $<I>$ represents the average intensity, and $i$ counts through all symmetry-related reflections 
of RRs, including the OmpR and $\mathrm{PhoB}$ as the representative members [18]. To date, only a few structures of PhoP have been reported because it is difficult to crystallize the full length of RRs in this subfamily [19]. Thus, more detailed structure information is required to understand their functional mechanisms such as the conformational changes accompanying with phosphorylation of PhoP and to compare with known PhoP structures. To determine its structure, a truncated form of PhoP from E. faecalis containing the receiver domain (EfPhoP-RD) was constructed as the first step. Here, we report the

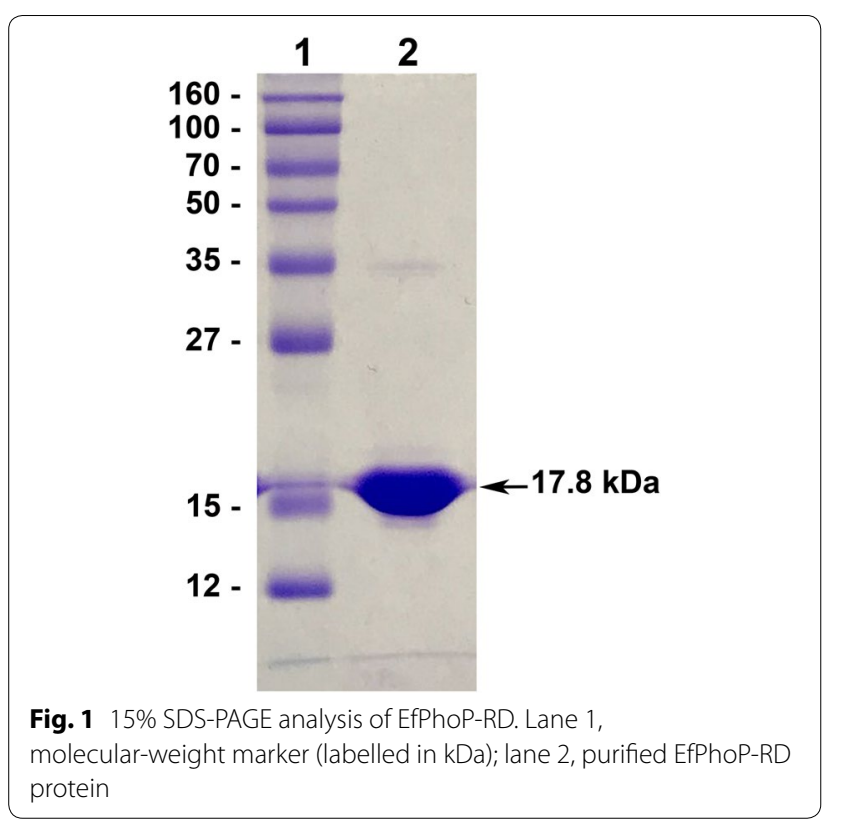

crystallization conditions and preliminary X-ray crystallographic analysis of EfPhoP-RD. Complete diffraction data sets was collected from apo-crystals at resolutions up to $3.5 \AA$.

\section{Materials and methods \\ Overexpression and purification of EfPhoP-RD protein}

The EfPhoP-RD gene was amplified from E. faecalis ATCC 29212 genomic DNA by polymerase chain reaction (PCR) using the forward and reverse primers. The primers contained respective modifications to treat suitable restriction endonucleases for insertion into the vector, where NdeI restriction site in the forward primer and the XhoI restriction site in the reverse primer are underlined in Table 1. The PCR-amplified DNA fragment was digested with NdeI and XhoI and was then inserted into the pET-28a expression vector (Novagen, USA). To enhance the solubilization of protein, the plasmid pEfPhoP-RD was generated to be expressed as EfPhoP-RD with hexa histidine-tag at the $\mathrm{N}$ - and $\mathrm{C}$-termini. Transformed E. coli BL21 (DE3) cells (Novagen, USA) harboring $\mathrm{pEfPhoP}-\mathrm{RD}$ were grown in Luria-Bertani medium with $50 \mu \mathrm{g} / \mathrm{ml}$ kanamycin at $25{ }^{\circ} \mathrm{C}$ to an optical density at $600 \mathrm{~nm}$ of $0.5-0.6$. Overexpression of recombinant EfPhoP-RD protein was induced by $0.5 \mathrm{mM}$ isopropyl- $\beta$ D-1-thiogalactopyrano-side and incubation at $18{ }^{\circ} \mathrm{C}$ for a further $12 \mathrm{~h}$. The cells were harvested by centrifugation at $5000 \mathrm{~g}$ for $20 \mathrm{~min}$ at $4{ }^{\circ} \mathrm{C}$.

The harvested cell pellets were resuspended in preequilibrium buffer A (0.02 M Tris- $\mathrm{HCl}, \mathrm{pH} 7.5,0.5 \mathrm{M}$ $\mathrm{NaCl}, 10 \%$ glycerol) adding $1 \mathrm{mM}$ phenylmethylsulfonyl

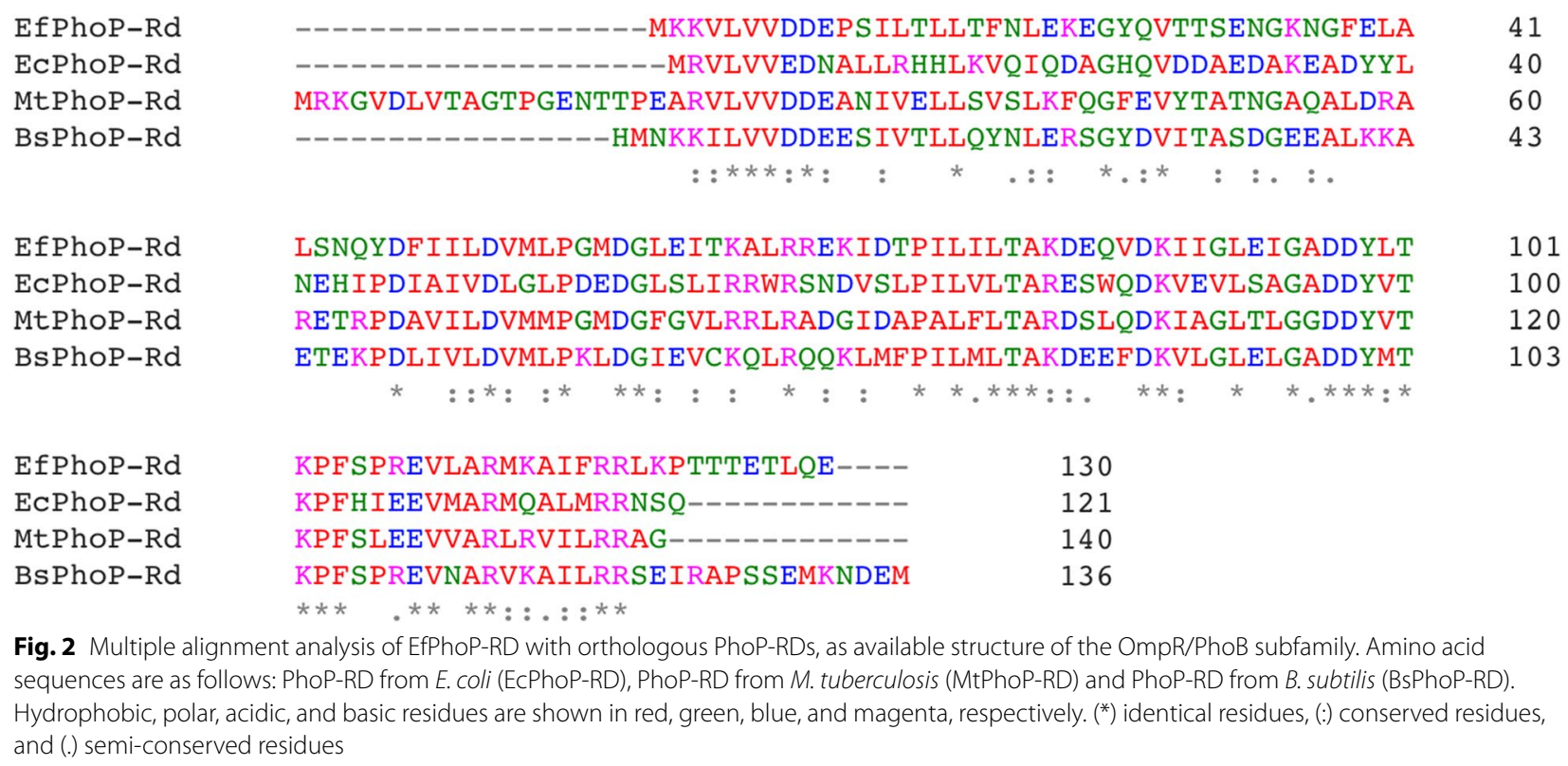

$\begin{array}{ll}\text { KPFSPREVLARMKAIFRRLKPTTTETLQE---- } & 130 \\ \text { KPFHIEEVMARMQALMRRNSQ------------ } & 121 \\ \text { KPFSLEEVVARLRVILRRAG------------- } & 140 \\ \text { KPFSPREVNARVKAILRRSEIRAPSSEMKNDEM } & 136\end{array}$

Fig. 2 Multiple alignment analysis of EfPhoP-RD with orthologous PhoP-RDs, as available structure of the OmpR/PhoB subfamily. Amino acid sequences are as follows: PhoP-RD from E. coli (EcPhoP-RD), PhoP-RD from M. tuberculosis (MtPhoP-RD) and PhoP-RD from B. subtilis (BsPhoP-RD). Hydrophobic, polar, acidic, and basic residues are shown in red, green, blue, and magenta, respectively. $\left(^{*}\right)$ identical residues, $(:)$ conserved residues, and (.) semi-conserved residues 
fluoride and ruptured by ultrasonication at $4{ }^{\circ} \mathrm{C}$. The crude lysate was centrifuged at $25,000 \mathrm{~g}$ for $20 \mathrm{~min}$ at $4{ }^{\circ} \mathrm{C}$. The supernatant was loaded onto a nickel $\left(\mathrm{Ni}^{2+}\right)$ charged HisTrap HP column (GE Healthcare, USA) equilibrated in buffer A. The bound EfPhoP-RD on the column was eluted with a linear gradient of elution buffer containing $0.02 \mathrm{M}$ Tris-HCl, pH 7.5, 0.5 M imidazole, $0.5 \mathrm{M} \mathrm{NaCl}$, $10 \%$ glycerol. The collected each fraction was confirmed by $15 \%$ SDS-PAGE, and subsequently purified by size exclusion chromatography on a HiLoad Superdex 200 column (GE Healthcare, USA) pre-equilibrated with buffer containing $0.02 \mathrm{M}$ Tris- $\mathrm{HCl} \mathrm{pH} 7.5,0.15 \mathrm{M} \mathrm{NaCl}$, $10 \%$ glycerol. The collected fractions containing EfPhoP$\mathrm{RD}$ were pooled and concentrated to $7.4 \mathrm{mg} / \mathrm{ml}$ using an Amicon Ultra-15 centrifugal filter device (Millipore, USA).

\section{Protein crystallization}

Preliminary screening for the crystallization of EfPhoP$R D$ was performed by the hanging-drop vapour-diffusion method in 96 -well microplates at $21{ }^{\circ} \mathrm{C}$ using various commercial screening kits such as Crystal Screen 1 and 2, PEGRx 1 and 2 (Hampton Research, USA), and Wizard Classic 1, 2, 3 and 4 (Rigaku Reagents Inc., USA). Initial crystals were obtained from two solutions as follows: the condition No. 30 of Crystal Screen 2 [0.1 M HEPES, $\mathrm{pH} 7.5,5 \%(v / v)$ (+/-)-2-methyl-2,4-pentanediol, $10 \%$ $(w / v)$ polyethylene glycol (PEG) 6000] and the condition No. 19 of PEGRx 2 [0.1 M Bis-Tris-propane, pH 9.0, $0.1 \mathrm{M} \mathrm{NaCl}, 25 \%(w / v)$ PEG 1500]. Optimization of EfPhoP-RD crystal was performed with the hanging-drop vapour-diffusion method in 24-well VDX plates (Hampton Research, USA) under conditions containing various PEGs and $\mathrm{pH}$ ranges; Each hanging drop was made by adding $1 \mu \mathrm{l}$ protein solution to $1 \mu \mathrm{l}$ reservoir solution to be a total volume of $2 \mu \mathrm{l}$ and was then equilibrated against $500 \mu \mathrm{l}$ reservoir solution.

\section{Collection and analysis of X-ray diffraction data}

For the collection of X-ray diffraction data in cryogenic condition, all EfPhoP-RD crystals were transferred to a cryoprotection solution with $25 \%(v / v)$ ethylene glycol added to each reservoir solution. The cryoprotected crystal was then rapidly cooled at $-180^{\circ} \mathrm{C}$ in a steam of liquid nitrogen. The sets of X-ray diffraction data were collected at beamline 7A in the Pohang Light Source (Pohang, South Korea) using an ADSC Quantum 270r CCD detector. A total range of $180^{\circ}$ was covered using $1^{\circ}$ oscillation and $5 \mathrm{~s}$ exposure/frame for thick plate-shape crystal, whereas a total range of $360^{\circ}$ was covered using $1^{\circ}$ oscillation and $2 \mathrm{~s}$ exposure/frame for thin rod-shape crystal. The wavelength of synchrotron radiation was

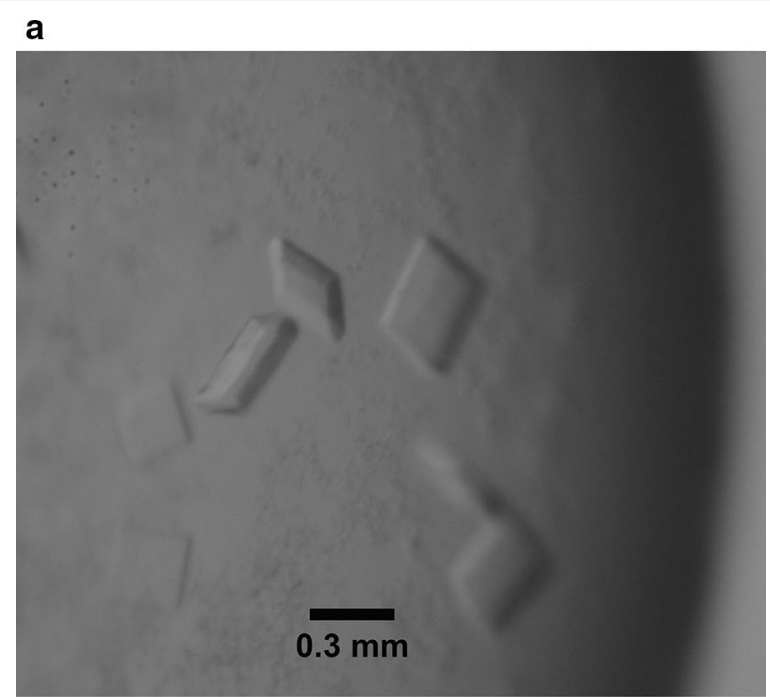

b

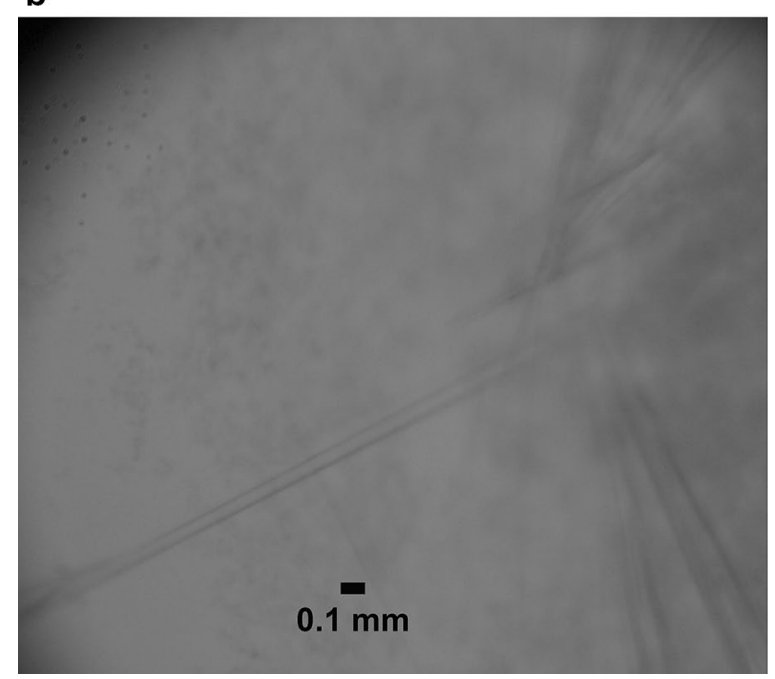

Fig. 3 Crystals of PhoP-RD from E. faecalis ATCC 29212. a

Thick plate-shape crystals of EfPhoP-RD; $\mathbf{b}$ Thin rod-shape crystals of EfPhoP-RD. The crystal dimensions of EfPhoP-RD are approximately $0.3 \times 0.3 \times 0.1 \mathrm{~mm}$ for thick plate-shape crystals and $0.1 \times 0.1 \times 0.8 \mathrm{~mm}$ for thin rod-shape crystals

$1,0000 \AA$. The crystal-to-detector distances of thick plateshape and thin rod-shape crystals were 450 and $350 \mathrm{~mm}$, respectively. All diffraction data sets were indexed to identify the unit cell and space group of the crystal, and then scaled after integration of the indexed data using the HKL2000 software package [20]. Detailed information on data collection is shown in Table 2.

\section{Results and discussion}

The gene encoding EfPhoP-RD (residue 1-130) from E. faecalis ATCC 29212 was successfully cloned into bacterial expression vector pET-28a. The recombinant 


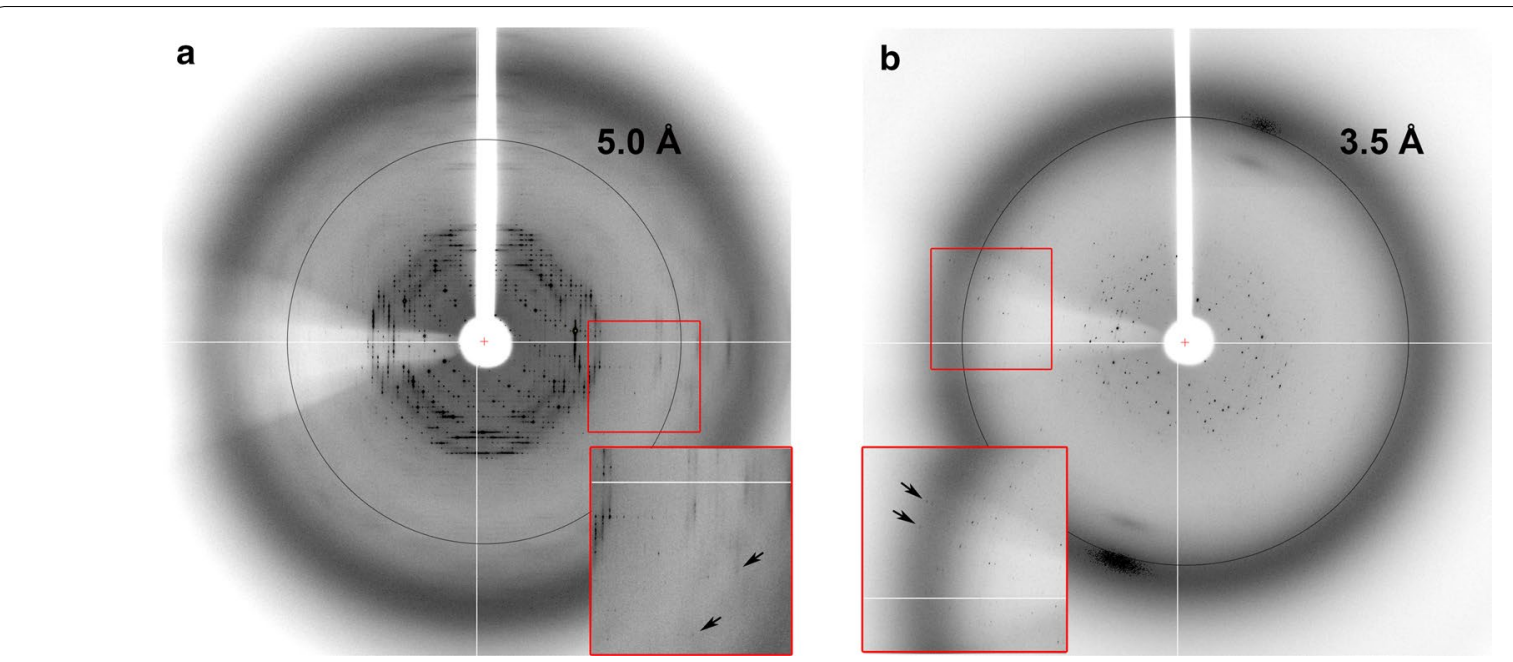

Fig. 4 X-ray diffraction patterns of EfPhoP-RD thick plate-shape $\mathbf{a}$ and thin rod-shape $\mathbf{b}$ crystals obtained using an ADSC Quantum 270r CCD detector. A resolution circle is shown at $5.0 \AA$ (a) and $3.5 \AA$ (b). The red boxes show the magnification of an area containing high-resolution spots indicated by the arrows

EfPhoP-RD protein containing 130 residues with a calculated molecular weight of $17.8 \mathrm{kDa}$, was overexpressed in E. coli BL21 (DE3). The purification of protein was carried out in two steps using a nickel-charged HisTrap $\mathrm{HP}$ and size exclusion columns. The purified EfPhoP-RD protein showed a single band on 15\% SDS-PAGE, with estimated purity over 95\% (Fig. 1). Amino acid sequence comparison of EfPhoP-RD was performed by multiple alignment analysis with receiver domains of PhoPs from E. coli (EcPhoP-RD) [21], Mycobacterium tuberculosis (MtPhoP-RD) [19] and B. subtilis (BsPhoP-RD) [22] as known structures of the OmpR/PhoB subfamily. EfPhoP$\mathrm{RD}$ (residue 1-130) shared low sequence identities with EcPhoP-RD (residue 1-121), MtPhoP-RD (residue 19-140), and BsPhoP-RD (residue 3-132) (36\%, 41\% and $54 \%$, respectively) (Fig. 2).

Based on initial crystallization conditions, crystals of EfPhoP-RD suitable size for X-ray diffraction were obtained within 4 days using two optimized reservoir solutions as follows. Several thick plate-shape and thin rod-shape crystals were obtained under reservoir solutions consisting of $0.1 \mathrm{M}$ Bis-Tris-propane, pH 9.0, 6-8\% $(w / v)$ PEG $6 \mathrm{~K}$ and 0.1 M HEPES, $\mathrm{pH} 7.5,4-5 \%(w / v)$ PEG $10 \mathrm{~K}$, respectively. Dimensions of thick plate-shape and thin rod-shape crystals were approximately $0.3 \times 0.3 \times 0.1 \mathrm{~mm}$ (Fig. 3a) and $0.1 \times 0.1 \times 0.8 \mathrm{~mm}$ (Fig. 3b), respectively. Thick plateshape crystal of EfPhoP-RD diffracted at a low resolution of $5.0 \AA$ (Fig. 4a). It was found to belong to the orthorhombic space group $P 2{ }_{1} 2_{1} 2_{1}$, with unit-cell parameters $a=188.632, b=187.706, c=197.488 \AA$. Assuming the presence of forty monomers per asymmetric unit, the
Matthews coefficient $V_{\mathrm{M}}$ value [23] was calculated to be $2.45 \AA^{3} \mathrm{Da}^{-1}$, with estimated solvent content of $49.87 \%$. On the other hand, thin rod-shape crystal of EfPhoP-RD diffracted to better than $3.5 \AA$ resolution (Fig. 4b). It was found to belong to the orthorhombic space group $C 222_{1}$, with unit-cell parameters $a=118.743, b=189.826$, $c=189.882 \AA$. Assuming the presence of twelve monomers per asymmetric unit, the Matthews coefficient $V_{\mathrm{M}}$ value [23] was calculated to be $2.50 \AA^{3} \mathrm{Da}^{-1}$, with estimated solvent content of $50.85 \%$.

Molecular replacement was attempted using MOLREP program [24] in the CCP4 [25] with the crystal structure of BsPhoP-RD (PDB ID: 1MVO) [22] indicating $54 \%$ sequence identity as a search model. However, our attempts could not provide a clear solution of structure for further refinement. This implies that the structure of EfPhoP-RD might contain a novel or different fold compared to other PhoP-RDs, although all data sets had low completeness and resolution. Therefore, the structure of EfPhoP-RD will be further determined by the MAD method [26] using selenomethionine substituted protein to solve the phase problem.

\section{Acknowledgements}

We would like to thank the staff of beamline 7A at the Pohang Accelerator Laboratory in South Korea for their assistance during X-ray data collection.

\section{Authors' contributions}

YCJ and KSL carried out experiments. YCJ and KSL designed experiments, analyzed data and wrote the manuscript. Both authors read and approved the final manuscript.

\section{Funding}

Not applicable. 
Availability of data and materials

Data sharing not applicable to this article as no datasets were generated or analyzed during the current study.

\section{Ethics approval and consent to participate}

Not applicable.

\section{Consent for publication}

Not applicable.

\section{Competing interests}

The authors declare that they have no competing interests.

Received: 7 October 2019 Accepted: 29 October 2019

Published online: 07 November 2019

\section{References}

1. Lamarche MG, Wanner BL, Crepin S, Harel J (2008) The phosphate regulon and bacterial virulence: a regulatory network connecting phosphate homeostasis and pathogenesis. FEMS Microbiol Rev 32(3):461-473

2. Ha S, Tran LS (2014) Understanding plant responses to phosphorus starvation for improvement of plant tolerance to phosphorus deficiency by biotechnological approaches. Crit Rev Biotechnol 34(1):16-30

3. Noh YD, Park HJ, Kim KR, Kim WI, Jung KY, Kim SU et al (2017) Contrasting effect of phosphate on phytoavailability of arsenic and cadmium in soils supporting medicinal plants. Appl Biol Chem 60(2):119-128

4. Chekabab SM, Harel J, Dozois CM (2014) Interplay between genetic regulation of phosphate homeostasis and bacterial virulence. Virulence 5(8):786-793

5. Santos-Beneit $F$ (2015) The Pho regulon: a huge regulatory network in bacteria. Front Microbiol 6:402

6. Wanner BL, Chang BD (1987) The phoBR operon in Escherichia coli K-12. J Bacteriol 169(12):5569-5574

7. Tommassen J, de Geus P, Lugtenberg B, Hackett J, Reeves P (1982) Regulation of the pho regulon of Escherichia coli K-12. cloning of the regulatory genes phoB and phoR and identification of their gene products. J Mol Biol. 157(2):265-274

8. Hulett FM, Lee J, Shi L, Sun G, Chesnut R, Sharkova E et al (1994) Sequential action of two-component genetic switches regulates the $\mathrm{PHO}$ regulon in Bacillus Subtilis. J Bacteriol 176(5):1348-1358

9. Parkinson JS (1993) Signal transduction schemes of bacteria. Cell 73(5):857-871

10. Hoch JA, Silhavy TJ (1995) Two-component signal transduction. ASM Press, Washington, D.C.

11. Novak R, Cauwels A, Charpentier E, Tuomanen E (1999) Identification of a Streptococcus pneumoniae gene locus encoding proteins of an ABC phosphate transporter and a two-component regulatory system. J Bacteriol 181(4):1126-1133
12. Glover RT, Kriakov J, Garforth SJ, Baughn AD, Jacobs WR Jr (2007) The two-component regulatory system senX3-regX3 regulates phosphatedependent gene expression in Mycobacterium smegmatis. J Bacteriol 189(15):5495-5503

13. Blanco AG, Sola M, Gomis-Ruth FX, Coll M (2002) Tandem DNA recognition by PhoB, a two-component signal transduction transcriptional activator. Structure 10(5):701-713

14. Galperin MY (2010) Diversity of structure and function of response regulator output domains. Curr Opin Microbiol 13(2):150-159

15. Murray BE (1990) The life and times of the Enterococcus. Clin Microbiol Rev 3(1):46-65

16. Teng F, Wang L, Singh KV, Murray BE, Weinstock GM (2002) Involvement of PhoP-PhoS homologs in Enterococcus faecalis virulence. Infect Immun 70(4):1991-1996

17. Arthur M, Depardieu F, Courvalin P (1999) Regulated interactions between partner and non-partner sensors and response regulators that control glycopeptide resistance gene expression in enterococci. Microbiology 145(Pt 8):1849-1858

18. Martinez-Hackert E, Stock AM (1997) Structural relationships in the OmpR family of winged-helix transcription factors. J Mol Biol 269(3):301-312

19. Menon S, Wang S (2011) Structure of the response regulator PhoP from Mycobacterium tuberculosis reveals a dimer through the receiver domain. Biochemistry 50(26):5948-5957

20. Otwinowski Z, Minor W (1997) Processing of X-ray diffraction data collected in oscillation mode. Methods Enzymol 276:307-326

21. Bachhawat P, Stock AM (2007) Crystal structures of the receiver domain of the response regulator PhoP from Escherichia coli in the absence and presence of the phosphoryl analog beryllofluoride. J Bacteriol 189(16):5987-5995

22. Birck C, Chen Y, Hulett FM, Samama JP (2003) The crystal structure of the phosphorylation domain in PhoP reveals a functional tandem association mediated by an asymmetric interface. J Bacteriol 185(1):254-261

23. Matthews BW (1968) Solvent content of protein crystals. J Mol Biol 33(2):491-497

24. Vagin A, Teplyakov A (2010) Molecular replacement with MOLREP. Acta Crystallogr D Biol Crystallogr 66(Pt 1):22-25

25. Winn MD, Ballard CC, Cowtan KD, Dodson EJ, Emsley P, Evans PR et al (2011) Overview of the CCP4 suite and current developments. Acta Crystallogr D Biol Crystallogr 67(Pt 4):235-242

26. Hendrickson WA, Horton JR, LeMaster DM (1990) Selenomethionyl proteins produced for analysis by multiwavelength anomalous diffraction (MAD): a vehicle for direct determination of three-dimensional structure. EMBO J 9(5):1665-1672

\section{Publisher's Note}

Springer Nature remains neutral with regard to jurisdictional claims in published maps and institutional affiliations.

\section{Submit your manuscript to a SpringerOpen ${ }^{\circ}$ journal and benefit from:}

- Convenient online submission

- Rigorous peer review

- Open access: articles freely available online

- High visibility within the field

- Retaining the copyright to your article

Submit your next manuscript at springeropen.com 